\title{
The Female Kindergarten Teachers' Relationships with Children with Insecure Attachment and Their Relationship with the Verbal Abilities of Those Children
}

\author{
Kawthar Mousa Mohammad Al Salhout and Prof. Jehan Wadiea Necola Mattar
}

\begin{abstract}
The present study aimed to explore the female kindergarten teachers' relationships with children with insecure attachment. It aimed to explore the relationship between such relationships and the verbal abilities of those children in University district, Amman. The sample consists from 110 children (i.e. 71 male children and 39 female children). Those children with insecure attachment with caregivers. The researchers adopted a descriptive correlative approach. They used a scale to identify the insecure attachment pattern. The latter scale was used to collect data from the primary caregivers of the children. The correlation coefficient values of the latter scale are within the range of (0.260- 0.631). The Cronbach alpha coefficient value of the latter scale is 0.903 . The researchers also used a scale for exploring the female kindergarten teachers' relationship with the children. The latter scale was used to collect data from the female kindergarten teachers. The correlation coefficient values of the latter scale are within the range of (0.552- 0.169). The Cronbach alpha coefficient value of the latter scale is 0.875 . The researchers used a scale for assessing the verbal abilities of each child. The correlation coefficient values of the latter scale are within the range of (0.187-0.624). The Cronbach alpha coefficient value of the latter scale is 0.875$)$. The level of the positive relationships between the female kindergarten teachers and the children with insecure attachment in Amman is moderate $(\mathrm{m}=2.600)$. The mean of the (closeness) dimension is high. The mean of the (conflict) demission and the mean of (dependency) dimension are low. 51.8\% of the sampled children have moderate verbal abilities level. There are statistically significant correlation between the kindergarten teachers' relationships with those children and the children's verbal abilities. It was found that $24.9 \%$ of the changes in the female kindergarten teacher-children relationship can be attributed to the children's verbal abilities

Keywords: Insecure attachment pattern, female kindergarten teachers' relationships, verbal abilities, kindergarten children

DOI: $10.7176 / \mathrm{JEP} / 11-33-17$

Publication date: November $30^{\text {th }} 2020$

\section{Introduction}

The first five years of one's life have a significant impact on one's personality and development. Therefore, many scholars specialized in the psychology field (e.g. Freud, Erikson and Bowlby) issued publications about those years of one's life. They wrote about the factors that affect this stage of life. Such factors include: genetic internal factors and environmental external factors. The genetic internal factors include: the child's attachment pattern and verbal abilities. The environmental external factors are represented in factors related to the child's surrounding environment. They include: the kindergarten enrolment and the extent of interacting socially with others.

The children who suffer from insecure attachment are more likely to face problems when enrolling them in the kindergarten than other children. That is because the former children are still not independent which is needed to adjust with the kindergarten environment. It is because those children aren't capable to trust the teacher and focus on the tasks instead of focusing on their internal needs. It is because those children have low self-confidence and self-efficacy levels. Thus, the children who suffer from insecure attachment are in need for getting additional support by the teachers.

The female kindergarten teachers must establish secure and comfortable environment for children. They must let children become familiar with the daily tasks. They must create secure and stable relationships with children (Golding et al., 2013). Such relationships can be created through providing children with psychological and emotional attention. Creating such relationships with children shall enable them to dedicate more effort and attention to doing the tasks assigned to them. It shall enable children to succeed in doing the tasks assigned to them. It shall make children feel satisfied with their social, emotional and cognitive achievements (Sierra, 2012).

The quality of the child-teacher relationship is affected by both parties, because it is a binary relationship. In other words, the teacher's beliefs and perceptions affect the quality of her relationship with the child. In addition, the child's verbal abilities and communication skills affect the quality of his relationship with the teacher (Rudasill et al., 2006). The quality of the child-teacher relationship is also affected by the child's emotional intelligence, cognitive development and social interaction levels (Lawrence, 1960). For instance, the teacher shall unintentionally prefer the child who respond much to her interactions and show high compliance with the guidelines of the tasks. She shall unintentionally prefer the child who interact with her much due to having good verbal abilities more than other children (Rudasill et al., 2006).
\end{abstract}


Having friendly child-teacher relationship shall significantly affect the child's development in various areas. Hence, it is necessary to identify the factors that affect the child-teacher relationship and take measures to enhance such relationship (Yoleri, 2016)

\section{Statement of the Problem and the Study's Questions:}

The present study aimed to shed a light on the children's relationship with their kindergarten's teachers . It aimed to explore the impact of the children's verbal abilities on such a relationship.

The female kindergarten teacher plays a significant role in making the child feel secure and comfortable in the surrounding environment. She makes the child feels so through interacting with him and enabling him to create positive relationships with others. The teacher's interaction with the child with insecure attachment is considered significant. For instance, it may change the way the child perceives people and the surrounding environment. It may make the child perceive the surrounding environment as a secure and safe environment (Ritblatt and Longstreth, 2019).

The female kindergarten teacher can make the child trust her through providing him with support when needed. The female kindergarten teacher can make the child experience emotional stability (Kris, 2018). Several studies were conducted about the female teacher-child relationship. They include: the ones conducted by Nur et al (2018), Lippard et al. (2018) and Commodari (2013). Based on the latter studies, the female teacher-child relationship may negatively or positively affect the child. The quality of the female teacher-child relationship affects the child's performance in doing school activities. It affects the child's motivation to engage in activities. It affects the child's social competence and emotional regulation abilities. It affects the child's abilities to adjust within the school environment.

In the light of the aforementioned information, the present study aimed to explore the female kindergarten teachers' relationship with the children suffering from insecure attachment. It aimed to explore the relationship between such relationships and the verbal abilities of those children in University District, Amman. This study was carried out to promote awareness among female kindergarten teachers about the factors affecting their relationships with their children. They conducted this study due to the significant role of female teachers in meeting the children's needs.

\section{The Study's Questions}

This study aimed to answer those questions

Q.1. What is the level of the positive relationships between the female kindergarten teachers and the children with insecure attachment in Amman?

Q.2. What is the verbal abilities level of the children suffering from insecure attachment in Amman?

Q.3. Is there any statistically significant relationship -at the statistical significance level of $(\alpha=0.05)$ - between the female kindergarten teachers' relationships with the children suffering from insecure attachment and the children's verbal abilities?

Q.4. To what degree can the targeted children's verbal abilities interpret the changes in the female kindergarten teacher-children relationship?

\section{The Study's Objectives:}

The present study aimed to explore the level of the positive relationships between the female kindergarten teachers and the children with insecure attachment in Amman. It aimed to explore the verbal abilities level of the children suffering from insecure attachment in Amman. It aimed to explore whether there is any statistically significant relationship - at the statistical significance level of $(\boldsymbol{\alpha}=0.05)$ - between the female kindergarten teachers' relationships with the children suffering from insecure attachment and the children's verbal abilities. It aimed to explore the degree to which the targeted children's verbal abilities interpret the changes in the female kindergarten teacher-children relationship

\section{The Study's Significance:}

Theoretical Significance: This theoretical framework and literature review of the present study can be used by researchers who want to conduct similar studies.

Practical Significance: The theoretical framework and literature review of the present study contribute to enriching the knowledge of female kindergarten teachers about the way meeting the needs of students. In addition, the scales of the present study can be used by the concerned professionals.

\section{Definition of Terms}

-Insecure attachment (Theoretical definition): It refers to a binary relationship in which the child is attached to the primary caregiver. In this relationship, the child has much love to the primary caregiver and depends on him/her. $\mathrm{He} / \mathrm{she}$ is always afraid of being rejected by the primary caregiver. He/she always desire to be close from the 
caregiver. He/she may feel angry when the caregiver doesn't meet his/her needs (Holmes, 1993, 67).

-Insecure attachment (Operational definition): It refers to the score that the child obtains on the insecure attachment scale. The latter scale was used to collect data from the primary caregivers of the children

The female teacher-children relationship (Theoretical definition): It refers to the female teacher's capability to understand the body language of the child which represents social and emotional messages. It involves the female teacher's capability to respond to such language effectively. It involves the female teacher's capability to provide the child with emotional support when needed. It involves how secure the child feels when interacting with his female teacher. It involves the degree to which the child feels capable of exploring the surrounding environment when having his female teacher around (Pianta and Sabol, 2012). It involves the female teachers' perception for the quality of her relationship with a specific child. It involves the interactive behavior of the child with the concerned female teacher. It involves the feelings of the child and the female teacher towards each other and perception about each other (Verschueren, 2012).

-The female teacher-children relationship (Operational definition): It refers to the score obtained by the female teacher on the scale used for exploring the female kindergarten teachers' relationship with the children. This scale aims to explore whether this relationship is based on (closeness, conflict, or dependency). It aims to explore how positive and influential the female teacher-children relationship is from the female teacher's perspective.

-The verbal ability (Theoretical definition): It refers to one's capability to express his thoughts in a verbal or written manner. It is affected by the amount of vocabulary that one has and one's capability to choose the right word on the right time and in the right place (Andrew et al., 2005).

The verbal ability (Operational definition): It refers to the degree that the child obtains on the verbal ability scale. It may be high, moderate or low.

\section{The Study's Limits and Limitations}

The present study targets the children who are enrolled in public kindergartens in Amman during the first semester of the year (2020/2021). The results of the present study can't be generalized, because they are limited to the sample type \& size, procedures, instrument, and limits. They can't be generalized, due the special circumstances at the time of conducting this study. To illustrate more, this study was conducted during the Coronavirus crisis.

\section{Theoretical framework:}

\section{First: Attachment pattern}

Children seek establishing relationships with others. Children's emotional relationships with the caregivers are very significant. That is because such relationships contribute to the protecting the child and providing the child with care. It is because such relationships contribute to the child survival. It is because such relationships serve as indicators of the psychological well-being of the teacher and the child (Bowlby, 1988).

Ainsworth used the term (secure base) in a study about attachment. The latter term refers to the support provided by the caregiver to the child (Holmes, 1993). Bowlby (1988) suggested that the caregiver-child relationship enables the child to create a cognitive mind map that represent cognitive associations (Underwood \& Rosen, 2011). The child's new relationship can be used for predicting the future behaviors of the child (Bowlby, 1982).

The attachment relationship manifests in the form of secure relationship that the child creates with his caregiver. Through having this secure relationship, the child shall be having enough courage to explore the surrounding environment and share his experiences with others. He shall feel comfortable even if his caregiver leaves the place he is present at (Ainsworth \& Bowlby, 1991). He is capable to trust others. He considers the world as a secure place (Kremmel, 2008).

However, the child may have insecure attachment to his caregiver. When having such attachment, the child shall not trust anyone except for his caregiver. He shall seek getting support from his caregiver. He shall consider his caregiver as the secure base. If the caregiver didn't respond as required to his needs, the child shall carry out behaviors to adjust himself with the risk. For instance, he might cry, show anger, withdraw himself or hold on to his caregiver (Ainsworth et al., 1978).

\section{Second: The female kindergarten teacher-child relationship}

Based on the attachment theory, Pianta conducted a comprehensive assessment for the female kindergarten teacher-child relationship. She suggested that the female kindergarten teacher is an alternative mother. She suggested that this teacher seeks adjusting the environment in order for the child to learn, engage in activities and enjoy emotional stability (Gregoriadis \& Tsigilis, 2008).

The female kindergarten teacher-child relationship may be based on (closeness, conflict or dependency) (Verschueren et al., 2012). The closeness-based relationship between the female kindergarten teacher and the child is represented in a warm relationship. It involves having open communication between both. In the latter relationship, the teacher shall have perceive her relationship with the child as a warm and positive relationship. 
The child shall perceive his relationship with the teacher as a source of love, knowledge, support and security. The latter relationship shall encourage the child to engage in the kindergarten activities (Birch \& Ladd, 1997). It shall positively affect the child. It reflects that there is direct contact between the child and the teacher (Tsigilis et al., 2017).

When having a conflict-based relationship between the female kindergarten teacher and the child, the teacher shall perceive this relationship as being negative and based on anger, and conflict (Birch \& Ladd, 1997). Having this relationship indicates that the teacher and the child have much disagreement with each other (Tsigilis et al., 2017).

When having a dependency-based relationship between the female kindergarten teacher and the child, the child shall be highly attached to the teacher. He shall ask the teacher for help even if he didn't need it. He shall avoid interacting with his peers. That is because he is less mature and have weaker ability to meet the social requirements. It is because he feels lonely and have negative feelings towards the kindergarten (Birch \& Ladd, 1997).

The dependency-based relationship between the female kindergarten teacher and the child is affected by the dominant culture in society. The way of perceiving this relationship is affected by culture more than the closenessbased relationship and the conflict-based relationship. It differs from one society's culture to another and from one's culture to another. For instance, the cultures that emphasize the significance of independency consider this relationship as a negative relationship. They consider the child's behavior in this relationship as being annoying. As for the cultures that emphasize the significance of the hierarchy of authority, they consider this relationship as being a positive and desired relationship (Tsigilis et al., 2017).

\section{Third: The verbal abilities of the kindergarten child:}

When the child becomes 5 years old, he shall have good ability of understanding the verbal language. The child's language development reflects the amount of vocabulary he possesses. It reflects the extent of mastering the language skills. It reflects the child's ability in using the grammar (Qatami, 2012).

The child develops gradually in terms of using language. He shall show creativity in the way of using language. For instance, he produces words that he has never heard. He uses various derivations of the same verb. He uses derivations in accordance with the surrounding context. He becomes capable of using language to communicate with others. He makes generalizations when using language. The older the child is, the more capable he shall be in using language. For instance, in later stage of childhood, he shall become capable of using sentences of complex structure. He shall become capable of using linking words in sentences and possess a major amount of vocabulary. He shall become capable of using prepositions, adverbs and conditional if (Younis, 2010). There are various indicators for assessing the child's language development. Such indicators include the amount of vocabulary he possesses and ability to define words. They include: ability to read, pronounce and use words efficiently (Qwai'esh, 2015)

\section{Previous Studies}

The researchers reviewed several studies that are related to the female teacher-child relationship and the child's verbal abilities. Some of those studies are published in Arabic and others are published in English. They are presented below:

Veríssimo et al. (2017) aimed to assess the teacher-child relationship in terms of the attachment pattern and child's verbal IQ. The sample consists from 52 preschoolers ( 25 males and 27 females). The ages of those children are within the range of 4-5 years. Those children were selected from Lisbon. The sample also consists from 4 teachers. The teachers' rating of child secure base behavior was significantly associated with both child attachment security and verbal IQ. Attachment security has a moderate impact on the relation between verbal IQ and teachers' ratings of secure base. The children with high verbal IQ level have better abilities to use their teacher as a secure base for exploration. The latter result applies whether the children have secure or insecure attachment

Masood (2017) aimed to assess the commination skills of kindergarten children. He aimed to explore the relationship between such skills and other variables. The sample consists from 425 mothers who have kindergarten children. It also consists from 265 children enrolled in public kindergartens in Riyad. It also consists from 160 children enrolled in private kindergartens in Riyad, Saudi Arabia. It was found that the sampled kindergarten children have good commination skills. There isn't any significant difference between the children's communication skills level which can be attributed to parents' academic qualification, gender, number of siblings and birth order. Mothers play a minor role in developing children's communication skills.

Yoleri (2016) aimed to explore ability to use children's mood and language skills for predicting the pattern of the female teacher-children relationship during the pre-school stage. The sample consists from 195 preschoolers who were selected from Turkey. It was found that there is a negative relationship between the teacher-child relationship score and the children's mood score. It was found that there is a positive relationship between the teacher-child relationship score and the children's verbal skills score. The greater the quality of the teacher-child 
relationship, the more developed the children's verbal skills are.

Commodari (2013) aimed to explore the teacher-child relationship during the pre-school stage. He aimed to assess the attention skills of preschoolers during learning activities in accordance with gender and age. The sample consists from 279 children (147 males and 132 females). 226 children have secure attachment and 53 children have insecure attachment. Those children were selected from Italy. The ages of those children are within the range of (4-5) years. It was found that the teacher-child relationship quality affects the child's attention skills. It was found that the children who have insecure attachment with their teacher show lower engagement in cooperative learning activities than other children. It was found that the children who have insecure attachment with their teacher show slower auditory and visual response than others in the learning activities.

Al-Rosan (2009) aimed to explore the impact of the parenting style and the female teacher-child relationship on the child' socio-emotional development. The sample consists from 325 children (161 males and 164 females). It also consists from 325 mothers and fathers, and 34 female teachers. The members of the sample were selected from Bani Kenani, Irbid. It was found that the most dominant female teacher-child relationships are the ones that are based on closeness and intimacy. Based on the multiple linear regression analysis, $52.29 \%$ of the changes in the child' socio-emotional development are attributed to the parenting style and the female teacher-child relationship.

Mccabe and Meller (2004) aimed to explore the relationship between language, and social competency. They aimed to explore the impact of language on social development. The sample consists from 71 children (18 males and 17 females). The ages of those children are within the range of (4-5) years. Regarding the children whose language skills are poor, they show lower social development than the other children. The latter result was confirmed through the parents scale and the teacher scale. Through the peers' scale, there aren't significant differences between the children whose verbal skills are excellent and the children whose verbal skills are poor. There aren't significant differences between the children who suffer from poor language skills and other children in terms of language skills. That means that some language skills are influenced by context and situations.

The aforementioned studies aimed to shed a light on the impacts of positive relationship between the female teacher and children. For instance, Commodari (2013) aimed to shed a light on the impact of the female teacherchild relationship on the child's attention while carrying out activities. He aimed to explore that among the children who have secure attachment and the children have insecure attachment. Similar to the study of Commodari (2013), the present study sheds a light on the female teacher-children relationship and target the children who have insecure attachment.

Similar to the studies of Yoleri (2016) and Veríssimo et al. (2017), the present study targets the children who have insecure attachment. Similar to the studies of Yoleri (2016) and Veríssimo et al. (2017), it sheds a light on the relationship between the female teacher-children relationship and children's verbal abilities. Similar to the studies of Mccabe and Meller (2004) and Masood (2017), it targets verbal abilities.

As far as the researchers know, the studies that target the female kindergarten teacher-children relationship are scarce. They include: the one conducted by Al-Rosan (2009). The latter researcher aimed to explore the impact of the parenting style and the female teacher-child relationship on the child' socio-emotional development. Contrary to the aforementioned studies, the present study aimed to explore the female kindergarten teachers' relationship with the children suffering from insecure attachment. It aimed to explore the relationship between such relationships and the verbal abilities of those children in Amman.

\section{Methodology \\ Approach}

A descriptive correlative approach has been adopted. That is because this approach fits with the nature of the study's questions. Through adopting the latter approach, the researchers used three scales. The first scale to identify the insecure attachment pattern. The second scale aims at exploring the female kindergarten teachers' relationship with the children. The third scale aims at assessing the verbal abilities of each child.

\section{Population and sample}

The study's population consists from all children who were enrolled in the public kindergarten in University District, Amman during the academic year (2020/2021). It consists from 952 children (462 males and 490 females). 24 kindergarten are targeted in the present study. These numbers are cited from the statistical data published by Queen Rania Center for Education and Information Technology. The latter center is affiliated with the Ministry of Education.

To meet the study's goals, the researchers used the following scales:

First: The attachment pattern scale

The initial version of this scale consists from 29 items. It sheds a light on several dimension (i.e. the child's response to being separated from his caregiver and reunion with him, the way the child acts with his adult family 
members, and the way the child acts with adult strangers).

\section{The validity of the attachment pattern scale}

The content validity of this scale was checked through passing the initial version of this scale to several experts. Those experts include experts who are specialized in English language and experts who are specialized in educational psychology. The experts who are specialized in English language were asked to assess the scale in terms of language and clarity. The experts who are specialized in educational psychology were asked to assess the scale in terms of relevancy to the goals. All the experts were asked to make addition, deletions and changes where needed.

Some of the items were re-drafted in the light of the experts' comments. The scale forms were passed to an exploratory sample. The latter sample consists from 50 caregivers at kindergarten. That was done to explore the psychometric characteristics of the scale.

\section{Discrimination Index}

Discrimination Index is represented in the correlation coefficient values. The correlation coefficient values of the attachment pattern scale represent the construct validity. They are within the range of $(0.260-0.631 * *)$. They are statistically significant at the statistical significance of 0.05 . The cut off point value on this scale is 76 . Thus, the ones who have insecure attachment are the ones who get 76 scores or more on the scale.

\section{Reliability of the attachment pattern scale}

To measure the reliability of this scale, Cronbach alpha coefficient value is calculated. It is 0.903 .

The rating categories of the attachment pattern scale

The five point Likert scale was adopted. It consists from five rating categories. Those categories are: always, often, sometimes, rarely and never. The scores of those categories are: 5, 4, 3, 2 and 1 respectively. The final version of the attachment pattern scale consists from 28 items. The scores of respondents on this scale are within the range of (140-28).

Second: The female kindergarten teachers-children relationship scale

This scale consists from 28 items. It aims to explore the way the female kindergarten teachers perceive their relationships with children.

The validity of the female kindergarten teachers-children relationship scale.

The content validity of this scale was checked through passing the initial version of this scale to several experts. Those experts include experts who are specialized in English language and experts who are specialized in educational psychology. The experts who are specialized in English language were asked to assess the scale in terms of language and clarity. The experts who are specialized in educational psychology were asked to assess the scale in terms of relevancy to the goals. All the experts were asked to make addition, deletions and changes where needed.

Some of the items were re-drafted in the light of the experts' comments. The scale forms were passed to an exploratory sample. The latter sample consists from 50 kindergarten children who don't belong to the actual sample of the study.

Discrimination Index

Discrimination Index is represented in the correlation coefficient values. The correlation coefficient values of the female kindergarten teachers-children relationship scale represent the construct validity. They are within the range of $(0.169-0.522 * *)$. They are statistically significant at the statistical significance of 0.05 .

Reliability of the female kindergarten teachers-children relationship scale

To measure the reliability of this scale, Cronbach alpha coefficient value is calculated. It is 0.767 .

The rating categories of the female kindergarten teachers-children relationship scale

The five point Likert scale was adopted. The final version of this scale consists from 26 items. It sheds a light on the following dimensions:

1)-Closeness: The scores of the items related to this dimension are within the range of 10-50 scores. Having a high score in this regard indicates that the child perceives the teacher as a source of knowledge and love. It indicates that there's warmth and open communication in this relationship

2)-Conflict: The scores of the items related to this dimension are within the range of 11-55 scores. Having a high score in this regard indicates that there is much conflict and anger in the relationship

3)- Dependency: The scores of the items related to this dimension are within the range of 5-25 scores. Having a high score in this regard indicates that depends much on the teacher.

The total score $=(55+11)$ - $($ the total score of the conflict dimension $)+($ the total score of the closeness dimension $)$ $+(25+5)-$ the total score of the dependency dimension. The total scores reflects how positive and influential the female kindergarten-children relationship is.

Third: The verbal abilities scale 
This scale was developed based on the three-stratum theory. It was developed after reviewing an Arabic language dictionary, kindergarten curricula and several studies. It sheds a light on the following dimensions: (picture vocabulary dimension, acronyms dimension, synonyms dimension and homonyms dimension).

\section{The validity of the verbal abilities scale}

The content validity of this scale was checked through passing the initial version of this scale to several experts. Those experts include experts who are specialized in English language and experts who are specialized in educational psychology. The experts who are specialized in English language were asked to assess the scale in terms of language and clarity. The experts who are specialized in educational psychology were asked to assess the scale in terms of relevancy to the goals. All the experts were asked to make addition, deletions and changes where needed.

Some of the items were re-drafted in the light of the experts' comments. The scale forms were passed to an exploratory sample. The latter sample consists from 50 kindergarten children who don't belong to the actual sample of the study.

\section{Discrimination Index}

Discrimination Index is represented in the correlation coefficient values. The correlation coefficient values of the verbal abilities scale represent the construct validity. They are within the range of $\left(0.187-0.624^{* *}\right)$. They are statistically significant at the statistical significance of 0.05 .

Reliability of the verbal abilities scale

To measure the reliability of this scale, Cronbach alpha coefficient value is calculated. It is 0.875 .

\section{The rating categories of the verbal abilities scale}

The right answer on the item related to picture vocabulary dimension gets one score and the wrong answer in this regard gets zero. The sample applies to any item related to the acronyms dimension, synonyms dimension and homonyms dimension. The final version of this scale consists from 36 items. The total scores on this scale are 45 scores. The verbal abilities levels are classified into high, moderate and low levels. The scores on children on this are within the range of 7-35 scores.

\section{The means on this score are classified as follows:}

7-16: Low

17-25: Moderate:

26-35: High

Statistical analysis

The following statistical methods are used to answer the study's questions:

-To answer the first question, means and standard deviations are calculated.

-To answer the second question, percentages and frequencies are calculated

- To answer the third question, Pearson correlation coefficient values are calculated

To answer the fourth question, the linear regression analysis was conducted

\section{The study's results}

\section{Results related to the first question:}

Q.1. What is the level of the positive relationships between the female kindergarten teachers and the children with insecure attachment in Amman?

To answer this question, means and standard deviations are calculated. They are presented in the table below Table (1): Means and standard deviations for identifying the level of the positive relationships between the female kindergarten teachers and the children with insecure attachment in Amman

\begin{tabular}{|l|l|l|l|l|l|}
\hline Rank & No. & Dimension & Mean & Std. & Level \\
\hline 1 & 1 & Closeness & 3.72 & .664 & High \\
\hline 2 & 3 & Conflict & 1.98 & .695 & Low \\
\hline 3 & 2 & Dependency & 1.87 & .676 & Low \\
\hline 4 & 4 & Overall & 2.60 & .379 & Moderate \\
\hline
\end{tabular}

Based on table (1), the overall mean is 2.60 which is moderate. The overall standard deviation is 0.379 . Thus, the level of the positive relationships between the female kindergarten teachers and the children with insecure attachment in Amman is moderate. The mean of the closeness dimension is 3.72 which is ranked first and high. The standard deviation of the latter dimension is 0.664 . The mean of the conflict dimension is 1.98 which is ranked second and low. The standard deviation of the latter dimension is 0.695 . The mean of the dependency dimension is 1.87 which is ranked last and low. The standard deviation of the latter dimension is 0.676 . The overall moderate level may be attributed to the fact that the female teacher-child relationship is affected by the child's characteristics 
of the female teacher's characteristics. For instance, the female teacher's sensitivity and perception about the child affect such a relationship. In addition, the child's verbal abilities affect such a relationship (Rudasill et al., 2006). In addition, there are several factors that affect such a relationship and aren't addressed through the present study. Such factors include: the child's mood, self-organization skills, and socio-economic conditions. They include: the classroom environment and the child-parents relationship (Spilt, 2010).

Having a high closeness level between the female teachers and the children with insecure attachment indicates that the teachers exerted much effort to meet the needs of those children. That's suggested by the attachment theory (Spilt, 2018). The results in this regard are consistent with the result concluded by Al-Rosan (2009). The latter researcher found that the most dominant female teacher-child relationships are the ones that are based on closeness

Results related to the second question

Q.2. What is the verbal abilities level of the children suffering from insecure attachment in Amman?

To answer this question, frequencies and percentages are calculated. They are presented in the table below

Table (2): The frequencies and percentages for exploring the verbal abilities level of the children suffering from insecure attachment in Amman.

\begin{tabular}{|l|l|l|l|}
\hline No. & Level & Frequency & Percentage \\
\hline 1 & High & 28 & $25.5 \%$ \\
\hline 2 & Moderate & 57 & $51.8 \%$ \\
\hline 3 & Low & 25 & $22.7 \%$ \\
\hline
\end{tabular}

Based on table (2), 28 children have high verbal abilities level. They represent $25.5 \%$ of the sample. 57 children have moderate verbal abilities level. They represent $51.8 \%$ of the sample. 25 children have low verbal abilities level. They represent $22.7 \%$ of the sample. Thus, most of the sampled children have moderate verbal abilities level. The verbal abilities level of 5 year old children reflects the amount of vocabulary they possess. It reflects their abilities to comply with grammatical rules (Qatami, 2012).

Having differences between children in terms of verbal abilities is considered something normal. It is attributed to having differences in terms of the speed of developing in language-related areas. It can be attributed to environmental factors and personal factors (Al-Otoom, 2010). The results in this regard are consistent with the results concluded by Masood (2017). The latter researcher found that the sampled kindergarten children have good commination skills. He also found that mothers play a minor role in developing their children's communication skills.

Results related to the third question:

Q.3. Is there any statistically significant relationship -at the statistical significance level of $(\boldsymbol{\alpha}=0.05)$ - between the female kindergarten teachers' relationships with the children suffering from insecure attachment and the children's verbal abilities?

To answer this question, Pearson correlation coefficient values are calculated. They are presented in the table below

Table (3): The Pearson correlation coefficient values for exploring the relationship between the female kindergarten teachers' relationships with the children suffering from insecure attachment and the children's verbal abilities

\begin{tabular}{|l|l|}
\hline Dimension & Pearson correlation coefficient value \\
\hline Closeness & $.840^{* *}$ \\
\hline Conflict & $-.604^{* *}$ \\
\hline Dependency & $-.337^{* *}$ \\
\hline Overall & $.499^{* *}$ \\
\hline
\end{tabular}

Based on table (3), there is a statistically significant relationship -at the statistical significance level of ( $\boldsymbol{\alpha}$ $=0.05)$ - between the female kindergarten teachers' relationships with the children suffering from insecure attachment and the children's verbal abilities. That is because the overall Pearson correlation coefficient is 0.499 .

The Pearson correlation coefficient value of the closeness dimension is 0.840 . The Pearson correlation coefficient values of conflict and dependency are $-.604 * *$ and $-.337 * *$ respectively. They are negative values. They are statistically significant at the statistical significance level of $(\boldsymbol{\alpha}=0.05)$. The values in table (3) indicate that having a positive female kindergarten-child relationship shall improve the child' verbal abilities and vice versa. They indicate that having a female kindergarten-child relationship that is based on conflict and dependency shall negatively affect the child' verbal abilities and vice versa. The results in this regard may be attributed to the fact that the child's verbal abilities during the beginning of the academic year affect the quality of his interaction and communication with the teacher and colleagues. The quality of such interaction and communication shall also affect the development of the child's verbal abilities (Maldonado et al., 2017). The results in this regard are consistent with the results reached by Yoleri (2016). The latter researcher found that there is a positive relationship between the teacher-child relationship score and the children's verbal skills score. He found that the greater the quality of the teacher-child relationship, the more developed the children's verbal skills are. 
Results related to the fourth question:

Q.4. To what degree can the targeted children's verbal abilities interpret the changes in the female kindergarten teacher-children relationship?

The linear regression analysis was conducted to explore the degree to which the targeted children's verbal abilities can interpret the changes in the female kindergarten teacher-children relationship. Table (4) presents the results of this analysis

Table (4): The results of the linear regression analysis to explore the degree to which the targeted children's verbal abilities can interpret the changes in the female kindergarten teacher-children relationship

\begin{tabular}{|c|c|c|c|c|c|c|c|c|c|}
\hline \multirow[t]{2}{*}{ Model } & \multicolumn{2}{|c|}{$\begin{array}{l}\text { Non-standard coefficient } \\
\text { values }\end{array}$} & \multirow[t]{2}{*}{$\begin{array}{l}\text { Beta } \\
\text { value } \beta\end{array}$} & \multirow[t]{2}{*}{$\mathrm{T}$ value } & \multirow[t]{2}{*}{ p. } & \multirow[t]{2}{*}{$\mathrm{R}$} & \multirow[t]{2}{*}{$\mathrm{R}^{2}$} & \multirow[t]{2}{*}{$\mathrm{F}$ value } & \multirow{2}{*}{$\begin{array}{l}\mathrm{R} \\
\text { square } \\
\text { change }\end{array}$} \\
\hline & $\begin{array}{l}\text { Standard } \\
\text { error }\end{array}$ & $\begin{array}{l}\text { Regression } \\
\text { coefficient value }\end{array}$ & & & & & & & \\
\hline Constant & -27.521 & 8.200 & & -3.356 & .001 & \multirow[b]{2}{*}{.499} & \multirow[b]{2}{*}{.249} & \multirow[b]{2}{*}{35.854} & \multirow[b]{2}{*}{$.000 *$} \\
\hline $\begin{array}{l}\text { Verbal } \\
\text { abilities }\end{array}$ & .413 & .069 & .499 & 5.988 & $.000^{*}$ & & & & \\
\hline
\end{tabular}

Based on table (4), the regression coefficient values indicate that $24.9 \%$ of the changes in the female kindergarten teacher-children relationship can be attributed to the children's verbal abilities. This percentage is significant at the statistical significance level of $\boldsymbol{\alpha}=0.05$. The regression coefficient values are statistically significant and positive $(\beta=0.499),(T=5.988)$, and $(\mathrm{P}=0.000)$. The coefficient of determination value $\left(\mathrm{R}^{2}\right)$ is 0.249 . The results in this regard indicate that having excellent verbal abilities enabled children to form strong relationships of high quality with their female teachers. That is because having excellent verbal abilities shall enable the child to respond much to her interactions and show high compliance with the guidelines of the tasks. Thus, the teachers shall unintentionally prefer such children (Rudasill et al., 2006).

The child who has excellent verbal abilities shall be able to communicate well and frequently with the teacher in a verbal manner. Through having such communication, he shall ask for information about activities, claim for opportunities to play or ask for help in settling disputes with peers. Through having such communication, the teacher shall prefer such a child more than other children. She shall feel that her relationship with this child is positive and full of warmth. The results in this regard are consistent with the results concluded by Veríssimo et al. (2017). The latter researchers found that the children with high verbal IQ level have better abilities to use their teacher as a secure base for exploration. The latter result applies whether the children have secure or insecure attachment. The results in this regard are consistent with the result concluded by White (2016). The latter researcher found that the children's language skills affected their probabilities of getting emotional and educational support. He found that the children's language skills affected their interaction with teachers.

\section{Conclusion}

The level of the positive relationships between the female kindergarten teachers and the children with insecure attachment in Amman is moderate $(\mathrm{m}=2.600)$. The mean of the (closeness) dimension is high. The mean of the (conflict) demission and the mean of (dependency) dimension are low. 51.8\% of the sampled children have moderate verbal abilities level. There are statistically significant correlation between the kindergarten teachers' relationships with those children and the children's verbal abilities. It was found that $24.9 \%$ of the changes in the female kindergarten teacher-children relationship can be attributed to the children's verbal abilities.

The study's recommendations:

The researchers recommend:

1) Providing more attention by female kindergarten teachers and educators to the kindergarten students. The researchers recommends paying attention to the factors that may affect the ability of female kindergarten teachers to form positive relationships with children.

2) Conducting more studies about the variables that may affect the female kindergarten teachers-children relationship.

References Published in Arabic Language:

Al-Rosan, Ayoub (2009). The impact of the parenting style and the female teacher-child relationship on the child' socio-emotional development in kindergarten in Bani Kenani district. $\mathrm{PhD}$ dissertation. Yarmouk University. Available on Dar Al-Manthoomah Database (MD: 729422)

Al-E'toom, Adnan (2010). Cognitive psychology: Theory and application. $1^{\text {st }}$ edition. Amman. Dar AlMaseerah.

Qatami, Yousif (2012). The cognitive and language development of child. Amman. Al-Ahleye for Publication and Distribution

Qwai'esh, Naser (2015). The obstacles hindering the child from expressing himself verbally during early 
hildhood stage. Unpublished MA thesis. Abdelhamid Ibn Badis University of Mostaganem. Mostaganem. Algeria.

Masood, Amal (2018). The communication skills of the kindergarten child and their relationship with some variables. The Psychological and Educational Sciences Journal. 19(1), 329-305

Younis, Mohammad (2010). The child language: An empirical study targeting children in kindergartens and primary schools in Yousif Al-Sadeeq in Faiyum in the light of the modern language studies. Ain AlJam'ah Bookshop

References Published in English Language:

Ainsworth, M and Bowlby, J (1991), An ethological approach to personality development. American psychologist,46, (4), 333-341.

Ainsworth, M, Blehar, M, Waters, E, and Wall, S (1978), Patterns of attachment: A psychological study of the strange situation.New Jersey: Lawrence Erlbaum Associates.

Andrew, M, Cobb, C and Giamietro, P (2005), Verbalability and teacher effectiveness. Journal of TeacherEducation, 56 (4), 343-354.

Birch, S, and Ladd, G(1997), The Teacher-Child Relationship and Children's Early School Adjustment. Journal of School Psychology, 35 (1), 61-79.

Bowlby, J (1982), Attachment and loss: Attachment.(2 ${ }^{\text {nd }}$ ed., vol.1), New York : Basic Books.

Bowlby, J (1988), A SECURE BASE Parent-Child Attachment and Healthy Human Development, New York :Basic Books.

Commodari, E (2013), Preschool teacher attachment and attention skills. Spring plus, 673, retrieved October 29. 2019, from https://springerplus.springeropen.com/articles/10.1186/2193-1801-2-673

Golding, K, Durrant, E, Fain, J, Frost, A, Mills, C, Roberts, N, Templeton, S and Worrall, H (2013), Observing Children with Attachment Difficulties in School: A Tool for Identifying and Supporting Emotional and Social Difficulties in Children Aged 5-11,London: Jessica Kingsley

Holmes, J (1993), John Bowlby and attachment theory. London: Routledge .

Kris,D(2018),How to Build a Trauma-Sensitive Classroom Where All Learners Feel Safe,California : Report Magazine. https://www.kqed.org/mindshift/52566/how-to-build-a-trauma-sensitive-classroom-where-all

Kremmel ، L. (2008)، Preschool children's understanding of love: It's relationship with attachment Security and social competence (Doctoral dissertation). Retrieved from proQuest Dissertation and Theses database

Lawrence, J (1960), Language and Communication, New York.

Lippard, C, Paro, K, Rouse, H and Crosby, D (2018), A Closer Look at Teacher-Child Relationships and Classroom Emotional Context in Preschool. Child\&YouthCare Forum, 47, 1-21. retrieved October 29. 2019, from https://doi.org/10.1007/s10566-017-9414-1

McCabe, P, and Meller, P (2004),The relationship Between Language and Social Competence: How Language Impairment Affects Social growth. Psychology in the Schools, 41 (3), 313-321.

Nur, I, Arnas,Y, Abbak, B and Kale, M( 2018), Mother-Child and Teacher-Child Relationships and Their Associations with School Adjustment in Pre-school. EDUCATIONAL SCIENCES: THEORY \& PRACTICE, 18 (1), 201-220.

Ritblatt, S and Longstreth, S (2019), Understanding Young Children's Play Seeing Behavior Through the Lens of Attachment Theory. Young Children,74 (2), 78-84. www.naeyc.org/resources/permissions

Rudasill, K, Kaufman, S, Justice, L and Pence, K (2006), Temperament and Language Skills as Predictors of Teacher-Child Relationship Quality in Preschool. Early Education and Development, 17(2), $271-291$. retrieved October 29. 2019, from http://digitalcommons.unl.edu/edpsychpapers/130.

Sabola, T, and Piantab, R (2012), Recent trends in research on teacher-child relationships. Attachment \& Human Development, 14 (3), 213-231. http://www.tandfonline.com

Spilt, J (2010), Relationships between teachers and disruptive children in kindergarten: An exploration of different methods and perspectives, and the possibility of change, Amsterdam: University VAN Amsterdam.

Spilt, J, Vervoort, E, and Verschueren, K (2018), Teacher-child dependency and teacher sensitivity predict engagement of children with attachment problems. School Psychology Quarterly, 33 (3), 419-427. retrieved October 29. 2019 fromhttps://www.ncbi.nlm.nih.gov/pubmed/28956936

Tsigilis, N, Gregoriadis, A (2008), Measuring Teacher-Child Relationships in the Greek Kindergarten Setting: AValidity Study of the Student-Teacher Relationship Scale-Short Form. EARLY EDUCATION AND
DEVELOPMENT,
$19 \quad(5) \quad 816-835$
retrieved
October
29.
2019

fromhttp://dx.doi.org/10.1080/10409280801975826

Tsigilis, N, Gregoriadis, A, and Grammatikopoulos, V (2017), Evaluating the Student-Teacher Relationship Scale in the Greek educational. Research Papers in Education, 33 (4), 414-426.

Underwood, M and Rosen, L (Eds.) (2011), Social Development Relationships in Infancy, Childhood, and 
Adolescsnce, New York: Guilford press.

Verschueren,K and Koomen, H (2012), Teacher-child relationships from an attachment perspective. Attachment \& Human Development, 14 (3), 205-211. http://www.tandfonline.com.

Veríssimo, M, Torres, N, Silva, F, Fernandes, C, Vaughn, B, and Santos, A(2017), Children's Representations of Attachment and Positive Teacher-Child Relationships. Frontiers in psychology, 8 (2270),1-7.

White,K (2016), "My Teacher Helps Me" : Assessing Teacher-Child Relationships From the Child's Perspective. JOURNAL OF RESEARCH IN CHILDHOOD EDUCATION. 30 (1), 29-41. retrieved October 29. 2019 from http://dx.doi.org/10.1080/02568543.2015.1105333

Yoleri, S (2016),Teacher-child Relationships in Preschool Period: The Roles of Child Temperament and Language Skills. International Electronic Journal of Elementary Education, 9 (1), 210-224. 